Available online at www.banglajol.info

Bangladesh J. Sci. Ind. Res. 44(1), 11-30, 2009
BANGLADESH JOURNAL OF SCIENTIFIC AND INDUSTRIAL RESEARCH

E-mail: bjsir07@gmail.com

BCSIR

\title{
Assessment of Tannery Based Chromium Eco-toxicity through Investigating Regional Bio-concentration in Commercially Produced Chicken Eggs and their Physical Properties
}

\author{
A. M. M. Maruf Hossain*a , M. Shahidul Islam ${ }^{\mathrm{b}}$, M. Moklesur Rahman ${ }^{\mathrm{a}}$, M. Mustafa \\ Mamun $^{\mathrm{a}}$, M. Azizul Islam Kazi ${ }^{\mathrm{b}}$, and Syed Fazle Elahi ${ }^{\mathrm{a}}$ \\ ${ }^{a}$ Department of Soil, Water and Environment, Faculty of Biological Sciences, University of \\ Dhaka; ${ }^{b}$ Analytical Research Division, BCSIR Laboratories, Dhaka, Bangladesh.
}

\begin{abstract}
In Bangladesh, among the routes of chromium eco-toxicity, feeds and fertilizer production from tanned skin-cut wastes is the most direct one leading to food chain contamination. The tanning industries of Hazaribagh are processing some 220 metric tons of hide a day with an associated release of $600-1000$ $\mathrm{Kg}$ of tanned skin-cut waste (SCW) resulting from per ton processed hide. The SCW are protein-rich and are unscientifically used to produce protein-concentrates for poultry and fish feeds, and organic fertilizer. In view of the facts, a huge migration of chromium can happen into poultry products, fish and vegetables, and further bio-magnify into food chain. The target population of this phenomenon is also huge. Regional bio-concentration of chromium was investigated in commercially produced chicken eggs. Though the routes of distribution of this hugely produced protein-concentrates from SCW are unknown, eggs were sampled from Dhaka and its nearby other seven districts covering the central region of Bangladesh. Twelve eggs were randomly sampled from each district's egg stock market while albumen and yolk in each egg were studied separately for all studies. Out of the 192 samples from 96 eggs, dry weight basis mean chromium concentration was found to be $1.9016 \mathrm{ppm}$ with a sample standard deviation of 0.1502. The concentration levels were ranged from maximum of $19.8051 \mathrm{ppm}$ to undetectable levels. Single poultry egg was found to contain a mean chromium content of $23.3809 \mu \mathrm{g}$, which exceeds adequate daily dietary intake of children up to 8 years of age as well as corresponds to major part for other age groups. The region-wise physical properties of the eggs were also studied which included USDA size grading by whole weight at raw condition, whole weight ratio of raw condition to complete boiled condition, weight ratio of boiled shell-albumen-yolk, moisture content of albumen of boiled eggs, and moisture content of yolk of boiled eggs. The mean whole weights of eggs at raw condition were within the range of $39.9568 \mathrm{~g}$ ('peewee' as per USDA size grading) to 62.4047 $\mathrm{g}$ ('large' as per USDA size grading), the mean whole weight ratio of raw condition to complete boiled
\end{abstract}

* Corresponding author, E- mail: *Email: mueed_abd@yahoo.com 
condition ranged between " $1: 0.9704$ " to " $1: 0.9782$ ", the minimum weight ratio of boiled shell-albumen-yolk for all sample classes were ranged from " $1: 2.0714: 1.1549$ " to " $1: 4.8810: 2.0677$ " whereas the maximum weight ratio ranged from " $1: 3.1933: 1.4557$ " to " $1: 7.8084: 3.3818$ ", the mean moisture content of albumen of boiled eggs varied within the range of $82.8876 \%$ to $84.7636 \%$, and the mean moisture content of yolk of boiled eggs were between $50.6563 \%$ to $52.5124 \%$. These properties are of importance for local-level as well as basic researches on poultry.

Key words: Heavy metal bio-concentration, Ecotoxicology, Egg science

\section{Introduction}

Leather, a traditional export item in Bangladesh, enjoys a good reputation worldwide for their quality. This sector plays a significant role in the economy of Bangladesh in terms of its contribution to export and domestic market. In south-west part of Dhaka city, there is a tannery area occupying 25 hectares of land at Hazaribagh, where about $90 \%$ of tannery industries of Bangladesh are located. The tanning industries of Hazaribagh are processing some 220 metric tons of hide a day with an associated release of 600 - $1000 \mathrm{Kg}$ of tanned skin-cut waste (SCW) resulting from production of each ton processed rawhide or skin (Zahid, et al., 2004). The SCW are sliced cut pieces of hides produced at the end of some tanning processes to maintain a definite thickness. Being protein origin, these wastes are converted to protein-concentrate to be used as poultry feed, fish feed, and in production of organic fertilizers with some treatment. One of the major concerns of these activities is the heavy metals, especially chromium, used in the tanning processes. Large amounts of chrome powder and chrome liquor are used during tanning process. Some $47 \%$ collagen and $85 \%$ chemicals enter the waste streams as effluent (UNIDO, 2000). This means that some $53 \%$ of the collagen and $15 \%$ of the chemicals used are retained in the leather. Hossain, et al., (2007) reported that proteinconcentrates produced from SCW were found to contain chromium at levels as high as $2.49 \%$. This suggests that a huge migration of chromium can take place into poultry products, fish and vegetables through use of the protein-concentrates as feed ingredient, and further bio-magnify into food chain. The present study is performed to assess the nature and extent of the suspected ecotoxicity through investigating regional bioconcentration in poultry eggs.

Poultry plays a vital role in the economy of Bangladesh in recent years. The contribution by livestock is $3 \%$ of the total GDP (BBS, 2004) and nearly $10 \%$ of the agricultural GDP (Poultry Business Directory, 2007). As food poultry provides meat and egg. An egg is a good source of energy, protein, and fat. A $50 \mathrm{~g}$ weighing grade A chicken egg provides estimated values of $297 \mathrm{KJ}$ energy, $6 \mathrm{~g}$ 
protein, $5 \mathrm{~g}$ fat (1.5g saturated, $2.0 \mathrm{~g}$ monounsaturated, and $0.8 \mathrm{~g}$ polyunsaturated), and 190mg cholesterol (FAO, 2007b). Chicken eggs are the most commonly eaten eggs, and are highly nutritious (FAO, 2007a). They supply a large amount of complete, highquality proteins (which contains all essential amino acids for humans), and provide significant amounts of several vitamins and minerals, including vitamin A, riboflavin, folic acid, vitamin B6, vitamin B12, choline, iron, calcium, phosphorus and potassium.

\section{Status of commercial poultry egg production and its relation with SCW produced protein- concentrates}

From the beginning of early 1990's, production of layer and broiler has taken the shape of an industry. Government of Bangladesh has declared poultry as a thrust sector and classified it as agro-based industry. By 2005, about 110,800 different sized farms have been established in the country (BRAC, 2005). At present a total of 6 million people are working in poultry sector (Rahman, 2007). About 130 breeding farms are producing 6 million broiler Day Old Chicks and 0.2 million layer Day Old Chicks per week from 3 million broiler Parent Stock and 0.5 million layer Parent Stock (Poultry Business Directory, 2007).

As per FAO's statistics, between 1961 and 2000, annual world egg production rose by more than 3.5 times to reach about $55 \mathrm{mil}$ lion tons (all eggs, not just hen eggs); about $6 \%$ are hatching eggs. This large, linear increase was due to the rapid expansion in egg production of developing countries. Starting from a low base of 3.8 million tons in 1961, developing countries achieved an increase of egg production almost 10-fold, with growth mainly in Asia (Gillin, 2003).

In the year 2000, Asian countries alone contributed $81 \%$ of total egg production of developing countries. In fact, egg production of Asian developing countries (36261 thousand MT) was double that produced in developed countries in 2000 (Gillin, 2003).

The total requirement for poultry feed in the years 1995 and 2000 were 0.528 and 1.055 million tons, respectively which increased enormously in 2006 as 22.756 million tons. There are 54 large and medium industrial type feed mills in Bangladesh of which 9 feed mills produce more than $50 \%$ of total feed, and about $50 \%$ of the feedstuffs are being imported (Poultry Business Directory, 2007). In 2005, about $52 \%$ of poultry feed were produced by feed mills and the rests by the farmers themselves. In that year about $90 \%$ broiler feeds were produced by the feed mills, whereas only $18 \%$ layer feeds were produced by feed mills (Poultry Business Directory, 2007). This means, most of layer farmers have used hand mixed feeds which can certainly incorporate the large distribution of protein-concentrates produced from SCW. 


\section{Materials and Methods}

\section{Study area and sampling}

The study area constituted eight districts including capital Dhaka and covered the central portion of Bangladesh. Dhaka, Narsingdi, Kishoreganj, Mymensingh, Tangail, Gazipur, Narayanganj, and Munshigonj were selected for the study. Commercially produced poultry eggs were randomly sampled from each district's egg stock market. Twelve eggs were sampled from each district. In Dhaka, Gazipur, and Tangail's egg stock market both brown and white colored eggs were found. Six eggs from each type were sampled from these three districts. In the rest five districts only brown colored eggs could be found. The collected samples are classified district-wise, which are shown in Table I. and yolk were separately oven-dried at $80^{\circ} \mathrm{C}$ to remove all moisture. The samples were oven-dried unless the difference between two readings of weight was found negligible. Each of oven-died albumen and yolk were treated and analyzed separately.

The samples were prepared by using $\mathrm{HNO}_{3}$ $\mathrm{HClO}_{4}$ digestion (Kebbekus and Mitra, 1998). Since the samples were of organic origin with a very high organic content, $\mathrm{HNO}_{3}-\mathrm{HClO}_{4}$ digestion was preferred over the more common $\mathrm{HNO}_{3}$ extraction for the determination of heavy metals. This strongly oxidizing digestion decomposes organics quickly and efficiently.

\section{Sample analysis}

Analysis of all prepared samples was performed through atomic absorption spec-

Table I. District-wise classification of collected samples

\begin{tabular}{l|l|c}
\hline \multicolumn{1}{c|}{ Sampling districts } & \multicolumn{1}{c}{$\begin{array}{c}\text { ID for brown colored } \\
\text { egg samples }\end{array}$} & \multicolumn{1}{c}{$\begin{array}{c}\text { ID for white colored } \\
\text { egg samples }\end{array}$} \\
\hline Dhaka & Dhaka - Brown & Dhaka - White \\
Narsingdi & Narsingdi - Brown & - \\
Kishoreganj & Kishoreganj - Brown & - \\
Mymensingh & Mymensingh - Brown & - \\
Tangail & Tangail - Brown & Tangail - White \\
Gazipur & Gazipur - Brown & Gazipur - White \\
Narayanganj & Narayanganj - Brown & - \\
Munshigonj & Munshigonj - Brown & - \\
\hline
\end{tabular}

\section{Sample preparation}

The egg samples were boiled in deionized water and after complete boiling albumen trophotometry (AAS). BDH standard solution was used for preparation of chromium 
standard curve. Chromium determination was done in air-acetylene flame.

\section{Study of physical properties}

A total of five following physical properties were studied for the samples, which are listed in Table II.

Property 1: USDA size grading by whole weight at raw condition

Property 2: Whole weight ratio of raw condition to complete boiled condition

Property 3: Weight ratio of boiled shell-albumen-yolk

Property 4: Moisture content of albumen of boiled eggs

Property 5: Moisture content of yolk of boiled eggs
All of these properties could not have studied for all samples due to practical inconveniences.

Whole weight at raw condition was measured after cleaning and subsequent air-drying of external surface of the eggs. Then the eggs were boiled in beaker containing distilled water in controlled temperature using hot plate. The eggs were boiled in $100^{\circ} \mathrm{C}$ for 30 minutes. After complete boiling these were air-dried before taking another weight. Albumen and yolk were separated and weighed twice, first to determine the weight ratio of boiled shell-albumen-yolk, and again after slicing them separately for oven drying. The later measurement was used to determine the moisture contents of albumens and yolks as during slicing slight sample loss can occur. Samples were dried in $100^{\circ} \mathrm{C}$ in oven until significant variation in their weight detected.

Table II. Physical properties studied for classification of samples

\begin{tabular}{|c|c|c|c|c|c|c|}
\hline \multirow{2}{*}{$\begin{array}{c}\text { Sampling } \\
\text { districts }\end{array}$} & \multirow{2}{*}{ Sample ID } & \multicolumn{5}{|c|}{ Physical properties studied } \\
\hline & & Property 1 & Property 2 & Property 3 & Property 4 & Property 5 \\
\hline \multirow[t]{2}{*}{ Dhaka } & Dhaka - Brown & 3 & 3 & 3 & 3 & 3 \\
\hline & Dhaka - White & 3 & 3 & 3 & 3 & 3 \\
\hline Narsingdi & Narsingdi - Brown & 3 & 3 & 3 & 3 & 3 \\
\hline Kishoreganj & Kishoreganj - Brown & 3 & 3 & 3 & 3 & 3 \\
\hline Mymensingh & Mymensingh - Brown & 3 & 3 & 3 & 3 & 3 \\
\hline \multirow[t]{2}{*}{ Tangail } & Tangail - Brown & 3 & 3 & 3 & & \\
\hline & Tangail - White & 3 & 3 & 3 & & \\
\hline \multirow[t]{2}{*}{ Gazipur } & Gazipur - Brown & 3 & 3 & 3 & & \\
\hline & Gazipur - White & 3 & 3 & 3 & & \\
\hline Narayanganj & Narayanganj - Brown & 3 & 3 & & & \\
\hline Munshigonj & Munshigonj - Brown & 3 & 3 & & & \\
\hline
\end{tabular}


Table III. Average physical parameters for comparative regional bio-concentration

\begin{tabular}{l|c|c}
\hline \multicolumn{1}{c|}{ Physical parameter } & Average values & Standard error \\
\hline Whole weight of eggs, (g) & 55.5098 & 0.7590 \\
Shell: albumen: yolk ratio & $1: 4.8672: 1.9775$ & $\mathrm{NA}$ \\
Albumen raw weight, (g) & 34.4401 & - \\
Albumen moisture content, (\%) & 84.0603 & 0.1512 \\
Albumen dry weight, (g) & 5.4900 & - \\
Yolk raw weight, (g) & 13.9930 & - \\
Yolk moisture content,(\%) & 51.3656 & 0.1616 \\
Yolk dry weight, (g) & 6.8054 & - \\
\hline
\end{tabular}

Table IV. Calculation of regional chromium bio-concentration

\begin{tabular}{|c|c|c|c|c|c|c|c|}
\hline $\begin{array}{l}\text { Sample } \\
\text { class }\end{array}$ & $\begin{array}{l}\text { Sample } \\
\text { size and } \\
\text { standard } \\
\text { error }\end{array}$ & \begin{tabular}{|c|} 
Cr in egg \\
albumen, $\mu \mathrm{g} / \mathrm{g}$ \\
(dry weight)
\end{tabular} & $\begin{array}{c}\text { Cr in total } \\
\text { egg albumen } \\
\mu g\end{array}$ & $\begin{array}{c}\text { Cr in egg } \\
\text { yolk, } \mu \mathrm{g} / \mathrm{g} \\
\text { (dry } \\
\text { weight) }\end{array}$ & $\begin{array}{c}\mathrm{Cr} \text { in } \\
\text { total egg } \\
\text { yolk, } \mu \mathrm{g}\end{array}$ & \begin{tabular}{|c|} 
Total Cr in \\
each egg \\
(albumen \\
+yolk), $\mu \mathrm{g}$
\end{tabular} & $\begin{array}{l}\text { Concentration } \\
\text { in total edible } \\
\text { egg (albumen } \\
\text { +yolk), } \mu \mathrm{g} / \mathrm{g}\end{array}$ \\
\hline $\begin{array}{l}\text { Brown Egg } \\
\text { (Dhaka) }\end{array}$ & $\begin{array}{c}6 \\
\text { Std.Error }\end{array}$ & $\begin{array}{l}1.5621 \\
0.1950\end{array}$ & $\begin{array}{c}8.5759 \\
-\end{array}$ & $\begin{array}{l}1.1354 \\
0.2149\end{array}$ & $\begin{array}{c}7.7269 \\
-\end{array}$ & $\begin{array}{c}16.3028 \\
-\end{array}$ & $\begin{array}{c}1.3259 \\
-\end{array}$ \\
\hline $\begin{array}{l}\text { White Egg } \\
\text { (Dhaka) }\end{array}$ & $\begin{array}{c}6 \\
\text { Std.Error }\end{array}$ & $\begin{array}{l}2.0858 \\
0.6073\end{array}$ & $\begin{array}{c}11.4510 \\
-\end{array}$ & $\begin{array}{l}1.3410 \\
0.4133\end{array}$ & $\begin{array}{c}9.1260 \\
-\end{array}$ & $\begin{array}{c}20.5771 \\
-\end{array}$ & $\begin{array}{c}1.6736 \\
-\end{array}$ \\
\hline $\begin{array}{l}\text { Brown Egg } \\
\text { (Narsingdi) }\end{array}$ & $\begin{array}{c}12 \\
\text { Std.Error }\end{array}$ & $\begin{array}{l}1.0671 \\
0.2070\end{array}$ & $\begin{array}{c}5.8584 \\
-\end{array}$ & $\begin{array}{l}1.0383 \\
0.2597\end{array}$ & $\begin{array}{c}7.0660 \\
-\end{array}$ & $\begin{array}{c}12.9244 \\
-\end{array}$ & $\begin{array}{c}1.0512 \\
-\end{array}$ \\
\hline $\begin{array}{l}\text { Brown Egg } \\
\text { (Kishoreganj) }\end{array}$ & $\begin{array}{c}12 \\
\text { Std.Error }\end{array}$ & $\begin{array}{l}2.1827 \\
0.3379\end{array}$ & $\begin{array}{c}11.9830 \\
-\end{array}$ & $\begin{array}{l}1.2334 \\
0.1995\end{array}$ & $\begin{array}{c}8.3938 \\
-\end{array}$ & $\begin{array}{c}20.3764 \\
-\end{array}$ & $\begin{array}{c}1.6573 \\
-\end{array}$ \\
\hline $\begin{array}{l}\text { Brown Egg } \\
\text { (Mymensingh) }\end{array}$ & $\begin{array}{c}12 \\
\text { Std.Error }\end{array}$ & $\begin{array}{l}2.9545 \\
0.3636\end{array}$ & $\begin{array}{c}16.2202 \\
-\end{array}$ & $\begin{array}{l}1.9318 \\
0.3741\end{array}$ & $\begin{array}{c}13.1467 \\
-\end{array}$ & $\begin{array}{c}29.3669 \\
-\end{array}$ & $\begin{array}{c}2.3884 \\
-\end{array}$ \\
\hline $\begin{array}{l}\text { Brown Egg } \\
\text { (Tangail) }\end{array}$ & $\begin{array}{c}6 \\
\text { Std.Error }\end{array}$ & $\begin{array}{l}1.9104 \\
0.3401\end{array}$ & $\begin{array}{c}10.4881 \\
-\end{array}$ & $\begin{array}{l}1.7997 \\
0.3797\end{array}$ & $\begin{array}{c}12.2477 \\
-\end{array}$ & $\begin{array}{c}22.7358 \\
-\end{array}$ & $\begin{array}{c}1.8491 \\
-\end{array}$ \\
\hline $\begin{array}{l}\text { White Egg } \\
\text { (Tangail) }\end{array}$ & $\begin{array}{c}6 \\
\text { Std.Error }\end{array}$ & $\begin{array}{l}1.7281 \\
0.2592\end{array}$ & $\begin{array}{c}9.4873 \\
-\end{array}$ & $\begin{array}{l}0.6670 \\
0.2476\end{array}$ & $\begin{array}{c}4.5392 \\
-\end{array}$ & $\begin{array}{c}14.0265 \\
-\end{array}$ & $\begin{array}{c}1.1408 \\
-\end{array}$ \\
\hline $\begin{array}{l}\text { Brown Egg } \\
\text { (Gazipur) }\end{array}$ & $\begin{array}{c}6 \\
\text { Std.Error }\end{array}$ & $\begin{array}{l}2.3930 \\
0.4060\end{array}$ & $\begin{array}{c}13.1376 \\
-\end{array}$ & $\begin{array}{l}1.5584 \\
0.4111\end{array}$ & $\begin{array}{c}10.6055 \\
-\end{array}$ & $\begin{array}{c}23.7431 \\
-\end{array}$ & $\begin{array}{c}1.9311 \\
-\end{array}$ \\
\hline $\begin{array}{l}\text { White Egg } \\
\text { (Gazipur) }\end{array}$ & $\begin{array}{c}6 \\
\text { Std.Error }\end{array}$ & $\begin{array}{l}1.4699 \\
0.1971\end{array}$ & $\begin{array}{c}8.0698 \\
-\end{array}$ & $\begin{array}{l}1.9289 \\
0.4274\end{array}$ & $\begin{array}{c}13.1269 \\
-\end{array}$ & $\begin{array}{c}21.1967 \\
-\end{array}$ & $\begin{array}{c}1.7240 \\
-\end{array}$ \\
\hline $\begin{array}{l}\text { Brown Egg } \\
\text { (Narayanganj) }\end{array}$ & $\begin{array}{c}12 \\
\text { Std.Error }\end{array}$ & $\begin{array}{l}3.3685 \\
1.4602\end{array}$ & $\begin{array}{c}18.4931 \\
-\end{array}$ & $\begin{array}{l}2.2028 \\
0.2418\end{array}$ & $\begin{array}{c}14.9909 \\
-\end{array}$ & $\begin{array}{c}33.4840 \\
-\end{array}$ & $\begin{array}{c}2.7233 \\
-\end{array}$ \\
\hline $\begin{array}{l}\text { Brown Egg } \\
\text { (Munshigonj) }\end{array}$ & $\begin{array}{c}12 \\
\text { Std.Error }\end{array}$ & $\begin{array}{l}3.3878 \\
1.5026\end{array}$ & $\begin{array}{c}18.5990 \\
-\end{array}$ & $\begin{array}{l}1.2682 \\
0.2536\end{array}$ & $\begin{array}{c}8.6306 \\
-\end{array}$ & $\begin{array}{c}27.2296 \\
-\end{array}$ & 2.2146 \\
\hline
\end{tabular}




\section{Results and Discussion}

\section{Regional bio-concentration of chromium}

Some average physical parameters were estimated from all samples so that the regional bio-concentration of chromium can be determined in a comparative way. The results obtained from these investigation are given in Table III.

On the basis of the average dry weight, calculation of regional chromium bio-concentration is shown in Table IV.

The average statistics for all egg samples, irrespective of albumen and yolk separately counted are presented in Table V.

\section{Table V. Statistics of all egg samples}

\begin{tabular}{l|c}
\hline Total sample number & 192 \\
Mean concentration, $\mu \mathrm{g} / \mathrm{g}$ & 1.9016 \\
Std. error of mean & 0.1502 \\
Minimum, $\mu \mathrm{g} / \mathrm{g}$ & $\mathrm{ND}$ \\
Maximum, $\mu \mathrm{g} / \mathrm{g}$ & 19.8051 \\
Average total content in single egg, $\mu \mathrm{g}$ & 23.3809 \\
\hline
\end{tabular}

* = Not detected
The comparison between albumen and yolk for their likelihood to contain chromium is shown in Fig. 1.

\section{Region-wise physical properties of the eggs}

The "United States Department of Agriculture" grades chicken eggs by weight per dozen (USDA, 2000). Egg masses in Table 6 have been calculated on the basis of the USDA size grades.

Table VI. USDA size grades on basis of mass per egg

\begin{tabular}{l|l}
\hline \multicolumn{2}{c}{ Modern sizes (USA) } \\
\hline Size & \multicolumn{1}{c}{ Mass per egg } \\
\hline Jumbo & Greater than 2.5 oz. or 71g \\
Extra Large (XL) & Greater than 2.55 oz. or 64g \\
Large (L) & Greater than 2 oz. or 57g \\
Medium (M) & Greater than 1.75 oz. or 50g \\
Small (S) & Greater than 1.5 oz. or 43g \\
Peewee & Greater than 1.25 oz. or 35g \\
\hline
\end{tabular}

The physical properties with their respective statistics for individual sample classes are presented in Tables VII to XVII.

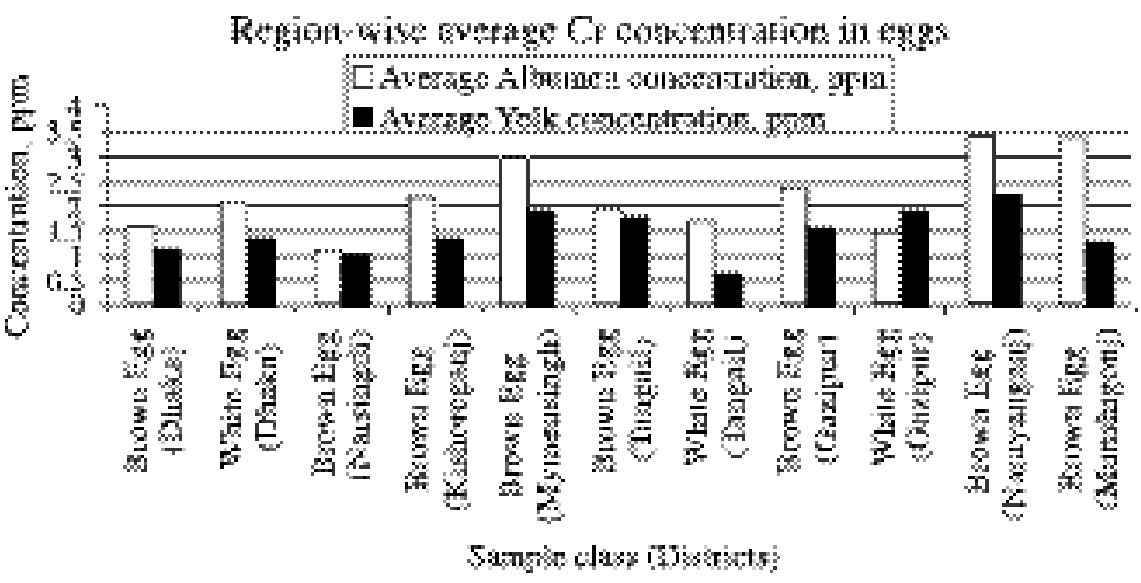

Fig. 1. Comparative chromium concentration in egg albumen and yolk 
Table VII. Physical properties of "Dhaka- Brown” class

\begin{tabular}{|c|c|c|c|c|c|c|}
\hline $\begin{array}{c}\text { Egg sample } \\
\text { sample } \\
\text { statistics }\end{array}$ & $\begin{array}{l}\text { Property 1: } \\
\text { weight (g) }\end{array}$ & $\begin{array}{l}\text { USDA } \\
\text { size } \\
\text { grade }\end{array}$ & $\begin{array}{c}\text { Property 2: whole } \\
\text { weight ratio of } \\
\text { raw to complete } \\
\text { boiled condition }\end{array}$ & $\begin{array}{c}\text { Property 3: } \\
\text { boiled shell } \\
\text { albumen-yolk } \\
\text { weight ratio }\end{array}$ & $\begin{array}{c}\text { Property 4: } \\
\text { boiled albu- } \\
\text { men moisture } \\
\text { content }(\%) \\
\end{array}$ & $\begin{array}{c}\text { Property 5: } \\
\text { boiled yolk } \\
\text { moisture } \\
\text { content (\%) }\end{array}$ \\
\hline $\mathrm{R}-1$ & 53.1718 & Medium & $1: 0.9678$ & $1: 6.0982: 2.2182$ & 82.0594 & 51.0165 \\
\hline R - 2 & 56.1305 & Medium & $1: 0.9692$ & 1: $5.8644: 1.9270$ & 84.0761 & 51.1205 \\
\hline $\mathrm{R}-3$ & 63.7801 & Large & $1: 0.9770$ & 1: $7.1942: 2.5219$ & 84.2837 & 50.6038 \\
\hline $\mathrm{R}-4$ & 54.421 & Medium & $1: 0.9722$ & $1: 4.5313: 1.6597$ & 84.4730 & 50.8725 \\
\hline R - 5 & 57.3433 & Large & $1: 0.9680$ & $1: 6.7456: 2.5732$ & 84.0844 & 50.5400 \\
\hline R - 6 & 61.2825 & Large & $1: 0.9681$ & $1: 7.4093: 2.4944$ & 84.5091 & 50.5520 \\
\hline $\begin{array}{l}\text { Minimum } \\
\text { value }\end{array}$ & 53.1718 & Medium & $1: 0.9678$ & $1: 4.5313: 1.6597$ & 82.0594 & 50.5400 \\
\hline $\begin{array}{l}\text { Maximum } \\
\text { value }\end{array}$ & 63.7801 & Large & $1: 0.9770$ & $1: 7.4093: 2.4944$ & 84.5091 & 51.1205 \\
\hline Mean & 57.6892 & Large & $1: 0.9704$ & NA* & 83.9143 & 50.7842 \\
\hline $\begin{array}{l}\text { Standard } \\
\text { error }\end{array}$ & 1.6694 & NA* & 0.0015 & NA & 0.3785 & 0.1034 \\
\hline
\end{tabular}

NA* = Not Applicable]

Table VIII. Physical properties of "Dhaka- Brown” class

\begin{tabular}{|c|c|c|c|c|c|c|}
\hline $\begin{array}{c}\text { Egg sample/ } \\
\text { sample } \\
\text { statistics }\end{array}$ & $\begin{array}{l}\text { Property 1: } \\
\text { weight (g) }\end{array}$ & $\begin{array}{l}\text { USDA } \\
\text { size } \\
\text { grade }\end{array}$ & $\begin{array}{l}\text { Property 2: whole } \\
\text { weight ratio of } \\
\text { raw to complete } \\
\text { boiled condition }\end{array}$ & $\begin{array}{c}\text { Property 3: } \\
\text { boiled shell } \\
\text { albumen-yolk } \\
\text { weight ratio }\end{array}$ & $\begin{array}{c}\text { Property 4: } \\
\text { boiled albu- } \\
\text { men moisture } \\
\text { content (\%) }\end{array}$ & $\begin{array}{c}\text { Property 5: } \\
\text { boiled yolk } \\
\text { moisture } \\
\text { content (\%) }\end{array}$ \\
\hline $\mathrm{R}-1$ & 60.7885 & Large & $1: 0.9688$ & $1: 5.6699: 2.0344$ & 83.6506 & 50.9005 \\
\hline R - 2 & 54.4185 & Medium & $1: 0.9714$ & $1: 5.9534: 2.8246$ & 85.4504 & 50.7258 \\
\hline $\mathrm{R}-3$ & 50.4281 & Medium & $1: 0.9762$ & $1: 6.0450: 2.3445$ & 84.8282 & 51.4818 \\
\hline $\mathrm{R}-4$ & 55.2353 & Medium & $1: 0.9710$ & $1: 4.5166: 1.5527$ & 83.1184 & 50.2009 \\
\hline R - 5 & 57.3005 & Large & $1: 0.9694$ & $1: 5.8753: 2.0129$ & 85.2394 & 51.0053 \\
\hline$R-6$ & 48.896 & Small & $1: 0.9702$ & $1: 5.5100: 1.9517$ & 84.8956 & 51.7757 \\
\hline $\begin{array}{l}\text { Minimum } \\
\text { value }\end{array}$ & 48.8960 & Small & $1: 0.9688$ & $1: 4.5166: 1.5527$ & 83.1184 & 50.2009 \\
\hline $\begin{array}{l}\text { Maximum } \\
\text { value }\end{array}$ & 60.7885 & Large & $1: 0.9762$ & $1: 6.0450: 2.3445$ & 85.4504 & 51.7757 \\
\hline Mean & 54.5112 & Medium & $1: 0.9716$ & NA & 84.5304 & 51.0150 \\
\hline $\begin{array}{l}\text { Standard } \\
\text { error }\end{array}$ & 1.7881 & NA & 0.0012 & NA & 0.3803 & 0.2278 \\
\hline
\end{tabular}


Table IX. Physical properties of "Narsingdi - Brown" class

\begin{tabular}{l|l|c|c|c|c|c}
\hline $\begin{array}{c}\text { Egg sample/ } \\
\text { sample } \\
\text { statistics }\end{array}$ & $\begin{array}{c}\text { Property 1: } \\
\text { weight (g) }\end{array}$ & $\begin{array}{c}\text { USDA } \\
\text { size } \\
\text { grade }\end{array}$ & $\begin{array}{c}\text { Property 2: whole } \\
\text { weight ratio of } \\
\text { raw to complete } \\
\text { boiled condition }\end{array}$ & $\begin{array}{c}\text { Property 3: boiled } \\
\text { shell albumen- } \\
\text { yolk weight ratio }\end{array}$ & $\begin{array}{c}\text { Property 4: } \\
\text { boiled albu- } \\
\text { men moisture } \\
\text { content (\%) }\end{array}$ & $\begin{array}{c}\text { Property 5: } \\
\text { boiled yolk } \\
\text { moisture } \\
\text { content (\%) }\end{array}$ \\
\hline $\mathrm{R}-1$ & 63.8716 & Large & $1: 0.9745$ & $1: 5.1597: 2.1951$ & 85.8635 & 49.9375 \\
$\mathrm{R}-2$ & 57.7092 & Large & $1: 0.9810$ & $1: 6.0377: 2.6406$ & 84.9249 & 50.4956 \\
$\mathrm{R}-3$ & 57.8982 & Large & $1: 0.9710$ & $1: 4.8810: 2.0677$ & 84.6685 & 51.0261 \\
$\mathrm{R}-4$ & 62.5003 & Large & $1: 0.9689$ & $1: 7.8084: 3.3818$ & 83.2821 & 50.3619 \\
$\mathrm{R}-5$ & 56.3723 & Medium & $1: 0.9681$ & $1: 5.7656: 2.3415$ & 84.9491 & 50.9399 \\
$\mathrm{R}-6$ & 67.6032 & Extra large & $1: 0.9702$ & $1: 5.5249: 2.1302$ & 85.3564 & 49.4025 \\
$\mathrm{R}-7$ & 60.3247 & Large & $1: 0.9706$ & $1: 5.9178: 2.3761$ & 85.2470 & 51.1561 \\
$\mathrm{R}-8$ & 61.9726 & Large & $1: 0.9726$ & $1: 6.7795: 2.6738$ & 85.3712 & 51.8413 \\
$\mathrm{R}-9$ & 60.6593 & Large & $1: 0.9699$ & $1: 6.2042: 2.7171$ & 84.5736 & 50.8230 \\
$\mathrm{R}-10$ & 62.4194 & Large & $1: 0.9730$ & $1: 5.8561: 2.1160$ & 84.4279 & 50.4320 \\
$\mathrm{R}-11$ & 60.2693 & Large & $1: 0.9705$ & $1: 7.5778: 2.8549$ & 83.9024 & 50.5394 \\
$\mathrm{R}-12$ & 58.7996 & Large & $1: 0.9680$ & $1: 7.6807: 2.9395$ & 84.5966 & 50.9208 \\
Minimum & & & & & & \\
value & 56.3723 & Mediun & $1: 0.9680$ & $1: 4.8810: 2.0677$ & 83.2821 & 49.4025 \\
Maximum & & & & & & \\
value & 67.6032 & Extra Large & $1: 0.9810$ & $1: 7.8084: 3.3818$ & 85.8635 & 51.8413 \\
Mean & 60.8666 & Large & $1: 0.9715$ & NA & 84.7636 & 50.6563 \\
Standard & & & & & & \\
error & 0.8904 & NA & 0.0010 & NA & 0.2013 & 0.1791 \\
\hline
\end{tabular}

\section{Mean physical properties of all samples}

The mean values for physical properties of all sample classes are presented in Table XVIII.

\section{Essentiality and toxicity of chromium}

Chromium is a mineral that humans require in trace amounts, although its mechanisms of action in the body and the amounts needed for optimal health are not well defined. Chromium as heavy metal has no adverse effect. The trivalent form of chromium is considered as essential for normal carbohydrate and lipid metabolism (NRC, 1980). Chromium is known to enhance the action of insulin (Mertz, 1998) a hormone critical to the metabolism and storage of carbohydrate, fat, and protein in the body. Little toxic effect is attributed to trivalent chromium when present in large concentrations. However, $\mathrm{Cr}$ (III) is ubiquitous in nature, occurring in air, water, soil, and biological materials. $\mathrm{Cr}(\mathrm{VI})$ has much higher toxicity in comparison to $\mathrm{Cr}(\mathrm{III})$, and the most important toxic 
Table X. Physical properties of "Kishoreganj - Brown" class

\begin{tabular}{|c|c|c|c|c|c|c|}
\hline $\begin{array}{l}\text { Egg sam- } \\
\text { ple/ } \\
\text { sample } \\
\text { statistics }\end{array}$ & $\begin{array}{l}\text { Property 1: } \\
\text { weight }(\mathrm{g})\end{array}$ & $\begin{array}{l}\text { USDA } \\
\text { size } \\
\text { grade }\end{array}$ & $\begin{array}{l}\text { Property 2: whole } \\
\text { weight ratio of } \\
\text { raw to complete } \\
\text { boiled condition }\end{array}$ & $\begin{array}{l}\text { Property 3: boiled } \\
\text { shell albumen- } \\
\text { yolk weight ratio }\end{array}$ & \begin{tabular}{|c|} 
Property 4: \\
boiled albu- \\
men moisture \\
content (\%)
\end{tabular} & $\begin{array}{c}\text { Property 5: } \\
\text { boiled yolk } \\
\text { moisture } \\
\text { content (\%) }\end{array}$ \\
\hline $\mathrm{R}-1$ & 60.4331 & Large & $1: 0.9692$ & $1: 4.0141: 2.0398$ & 84.7147 & 52.9912 \\
\hline $\mathrm{R}-2$ & 54.1158 & Medium & $1: 0.9711$ & $1: 2.9015: 1.3200$ & 83.6934 & 48.9653 \\
\hline $\mathrm{R}-3$ & 50.543 & Medium & $1: 0.9735$ & $1: 3.6885: 1.6984$ & 85.0742 & 53.6001 \\
\hline $\mathrm{R}-4$ & 59.4774 & Large & $1: 0.9685$ & $1: 2.4464: 1.4271$ & 84.2902 & 51.1880 \\
\hline$R-5$ & 53.8434 & Medium & $1: 0.9711$ & $1: 4.1202: 1.6948$ & 84.1460 & 53.2331 \\
\hline$R-6$ & 63.82 & Large & $1: 0.9775$ & $1: 3.7207: 1.6230$ & 84.6314 & 52.6694 \\
\hline $\mathrm{R}-7$ & 53.0333 & Medium & $1: 0.9707$ & $1: 4.4679: 1.8607$ & 85.6527 & 52.8032 \\
\hline $\mathrm{R}-8$ & 59.7496 & Large & $1: 0.9809$ & $1: 3.7479: 1.8190$ & 84.5351 & 53.5434 \\
\hline$R-9$ & 48.493 & Small & $1: 0.9744$ & $1: 4.0969: 1.5061$ & 83.8783 & 54.1786 \\
\hline$R-10$ & 67.388 & Extra large & $1: 0.9889$ & $1: 3.0995: 1.4257$ & 83.6152 & 51.1574 \\
\hline $\mathrm{R}-11$ & 66.9525 & Extra Large & $1: 0.9778$ & $1: 3.5853: 2.0774$ & 84.3932 & 53.2080 \\
\hline $\mathrm{R}-12$ & 49.3147 & Small & $1: 0.9744$ & $1: 4.1113: 2.0180$ & 83.7893 & 52.6114 \\
\hline $\begin{array}{l}\text { Minimum } \\
\text { value }\end{array}$ & 48.4930 & Small & $1: 0.9685$ & $1: 2.4464: 1.4271$ & 83.6152 & 48.9653 \\
\hline $\begin{array}{l}\text { Maximum } \\
\text { value }\end{array}$ & 67.3880 & Extra Large & $1: 0.9889$ & $1: 4.4679: 1.8607$ & 85.6527 & 54.1786 \\
\hline Mean & 57.2637 & Large & $1: 0.9748$ & NA & 84.3678 & 52.5124 \\
\hline $\begin{array}{l}\text { Standard } \\
\text { error }\end{array}$ & 1.9174 & NA & 0.0017 & NA & 0.1745 & 0.4133 \\
\hline
\end{tabular}

effects, after contact, inhalation, or ingestion of hexavalent chromium compounds include dermatitis, allergic and eczematous skin reactions, skin and mucous ulcerations, perforation of the nasal septum, allergic asthmatic reactions, bronchial carcinomas, gastro-enteritis, hepatocellular deficiency, and renal oligo-anuric deficiency (Baruthio, 1992).

\section{In vivo carcinogenicity of chromium}

Chromium occurs most commonly in valance states of $3+$ and $6+. \mathrm{Cr}^{3+}$ is the most stable oxidation state (Greenwood and Earnshaw, 1997) and presumably is the form in the food supply due to the presence of reducing substances in foods. Even a bolus dose of $5 \mathrm{mg} \mathrm{Cr}^{6+}$ was reduced to $\mathrm{Cr}^{3+}$ in 0.5 L of orange juice (Kuykendall et al., 1996), and endogenous reducing agents within the 
Table XI. Physical properties of "Mymensingh - Brown" class

\begin{tabular}{|c|c|c|c|c|c|c|}
\hline $\begin{array}{l}\text { Egg sam- } \\
\text { ple/ } \\
\text { sample } \\
\text { statistics }\end{array}$ & $\begin{array}{l}\text { Property 1: } \\
\text { weight }(\mathrm{g})\end{array}$ & $\begin{array}{l}\text { USDA } \\
\text { size } \\
\text { grade }\end{array}$ & $\begin{array}{l}\text { Property 2: whole } \\
\text { weight ratio of } \\
\text { raw to complete } \\
\text { boiled condition } \\
\end{array}$ & $\begin{array}{l}\text { Property 3: boiled } \\
\text { shell albumen- } \\
\text { yolk weight ratio }\end{array}$ & \begin{tabular}{|c|} 
Property 4: \\
boiled albu- \\
men moisture \\
content (\%) \\
\end{tabular} & $\begin{array}{c}\text { Property 5: } \\
\text { boiled yolk } \\
\text { moisture } \\
\text { content (\%) }\end{array}$ \\
\hline $\mathrm{R}-1$ & 54.2869 & Medium & $1: 0.9705$ & $1: 3.1665: 1.4392$ & 82.4655 & 50.9407 \\
\hline $\mathrm{R}-2$ & 49.2058 & Small & $1: 0.9726$ & $1: 2.0714: 1.1549$ & 83.3195 & 52.8795 \\
\hline$R-3$ & 54.7403 & Medium & $1: 0.9721$ & $1: 3.7493: 1.2290$ & 83.8288 & 52.4243 \\
\hline $\mathrm{R}-4$ & 56.5528 & Medium & $1: 0.9726$ & $1: 3.3299: 1.4300$ & 84.0992 & 51.7156 \\
\hline$R-5$ & 54.3853 & Medium & $1: 0.9734$ & $1: 2.9382: 1.3823$ & 80.7665 & 50.4200 \\
\hline $\mathrm{R}-6$ & 60.5077 & Large & $1: 0.9711$ & $1: 3.2820: 1.1829$ & 82.1882 & 51.1330 \\
\hline $\mathrm{R}-7$ & 55.6505 & Medium & $1: 0.9711$ & $1: 2.8930: 1.4356$ & 83.0736 & 50.7611 \\
\hline $\mathrm{R}-8$ & 55.1685 & Medium & $1: 0.9789$ & $1: 3.1411: 1.2366$ & 82.5688 & 51.0391 \\
\hline $\mathrm{R}-9$ & 52.1831 & Medium & $1: 0.9706$ & $1: 3.1602: 1.4058$ & 83.0579 & 51.3165 \\
\hline$R-10$ & 55.0537 & Medium & $1: 0.9720$ & $1: 3.3626: 1.5647$ & 82.6263 & 50.9080 \\
\hline $\mathrm{R}-11$ & 52.7628 & Medium & $1: 0.9721$ & $1: 3.2657: 1.4021$ & 82.9843 & 51.2608 \\
\hline$R-12$ & 58.1148 & Large & $1: 0.9717$ & $1: 2.8655: 1.2724$ & 83.6721 & 51.9305 \\
\hline $\begin{array}{l}\text { Minimum } \\
\text { value }\end{array}$ & 49.2058 & Small & $1: 0.9705$ & $1: 2.0714: 1.1549$ & 80.7665 & 50.4200 \\
\hline $\begin{array}{l}\text { Maximum } \\
\text { value }\end{array}$ & 60.5077 & Large & $1: 0.9789$ & $1: 3.7493: 1.2290$ & 84.0992 & 52.8795 \\
\hline Mean & 54.8844 & Medium & $1: 0.9724$ & NA & 82.8876 & 51.3941 \\
\hline $\begin{array}{l}\text { Standard } \\
\text { error }\end{array}$ & 0.8267 & NA & 0.0006 & NA & 0.2547 & 0.2072 \\
\hline
\end{tabular}

upper gastrointestinal tract and the blood also serve to prevent systemic uptake of $\mathrm{Cr}^{6+}$ (Kerger et al., 1997). But in their in vivo toxicity the intermediates of $\mathrm{Cr}^{5+}$ and $\mathrm{Cr}^{4+}$, or indirectly the reduced $\mathrm{Cr}^{3+}$ can be ultimately responsible.

In the carcinogenic behavior of chromium, chromate $\left(\mathrm{CrO}_{4}{ }^{2-}\right)$ (which is a strong oxidizing agent) is reduced intracellularly to $\mathrm{Cr}^{5+}$ inside biological system and reacts with nucleic acids and other cell components to produce mutagenic and carcinogenic effects on biological systems (Clark, 1994; McLean and Beveridge, 2001).

But Cohen and Costa (2000) mentioned about derived trivalent form of chromium to be ultimately responsible for the carcinogenic effect. The Cr (VI) ion is readily taken up into eukaryotic cells by anion-carrying proteins, after which it is reduced to $\mathrm{Cr}$ (III) 
Table XII. Physical properties of "Tangail - Brown" class

\begin{tabular}{|c|c|c|c|c|}
\hline $\begin{array}{c}\text { Egg sample/ } \\
\text { sample } \\
\text { statistics } \\
\end{array}$ & $\begin{array}{l}\text { Property 1: } \\
\text { weight (g) }\end{array}$ & $\begin{array}{l}\text { USDA size } \\
\text { grade }\end{array}$ & $\begin{array}{c}\text { Property 2: whole } \\
\text { weight ratio of raw to } \\
\text { complete boiled condition }\end{array}$ & $\begin{array}{l}\text { Property 3: boiled shell } \\
\text { albumen-yolk weight ratio }\end{array}$ \\
\hline $\mathrm{R}-1$ & 54.6955 & Medium & $1: 0.9747$ & $1: 3.0487: 1.2567$ \\
\hline$R-2$ & 51.7022 & Medium & $1: 0.9739$ & $1: 3.0213: 1.2868$ \\
\hline $\mathrm{R}-3$ & 54.6834 & Medium & $1: 0.9743$ & $1: 2.8551: 1.1622$ \\
\hline $\mathrm{R}-4$ & 49.9917 & Small & $1: 0.9729$ & $1: 3.1933: 1.4557$ \\
\hline$R-5$ & 54.6611 & Medium & $1: 0.9722$ & $1: 3.1671: 1.4640$ \\
\hline$R-6$ & 51.0989 & Medium & $1: 0.9760$ & $1: 2.9715: 1.3273$ \\
\hline $\begin{array}{l}\text { Minimum } \\
\text { value }\end{array}$ & 49.9917 & Small & $1: 0.9722$ & $1: 2.8551: 1.1622$ \\
\hline $\begin{array}{l}\text { Maximum } \\
\text { value }\end{array}$ & 54.6955 & Medium & $1: 0.9760$ & $1: 3.1933: 1.4557$ \\
\hline Mean & 52.8055 & Medium & $1: 0.9740$ & NA \\
\hline $\begin{array}{l}\text { Standard } \\
\text { error }\end{array}$ & 0.8677 & NA & 0.0005 & NA \\
\hline
\end{tabular}

Table XIII. Physical properties of "Tangail - White" class

\begin{tabular}{l|c|c|c|c}
\hline $\begin{array}{c}\text { Egg sample/ } \\
\text { sample } \\
\text { statistics }\end{array}$ & $\begin{array}{c}\text { Property 1: } \\
\text { weight (g) }\end{array}$ & $\begin{array}{c}\text { USDA size } \\
\text { grade }\end{array}$ & $\begin{array}{c}\text { Property 2: whole } \\
\text { weight ratio of raw to } \\
\text { complete boiled condition }\end{array}$ & $\begin{array}{c}\text { Property 3: boiled shell } \\
\text { albumen-yolk weight ratio }\end{array}$ \\
\hline $\mathrm{R}-1$ & 63.6611 & Large & $1: 0.9741$ & $1: 3.6539: 1.8125$ \\
$\mathrm{R}-2$ & 55.8108 & Medium & $1: 0.9768$ & $1: 3.3684: 2.0007$ \\
$\mathrm{R}-3$ & 57.7141 & Large & $1: 0.9761$ & $1: 3.3576: 1.9475$ \\
$\mathrm{R}-4$ & 60.7531 & Large & $1: 0.9760$ & $1: 3.5093: 1.7176$ \\
$\mathrm{R}-5$ & 57.3988 & Large & $1: 0.9746$ & $1: 2.9558: 1.5224$ \\
$\mathrm{R}-6$ & 55.2091 & Medium & $1: 0.9745$ & $1: 2.9529: 1.7272$ \\
$\begin{array}{l}\text { Minimum } \\
\text { value }\end{array}$ & 55.2091 & Medium & $1: 0.9741$ & $1: 3.6539: 1.8125$ \\
$\begin{array}{l}\text { Maximum } \\
\text { value }\end{array}$ & 63.6611 & Large & $1: 0.9768$ & $\mathrm{NA}$ \\
$\begin{array}{l}\text { Mean } \\
\text { Standard } \\
\text { error }\end{array}$ & 58.4245 & Large & $1: 0.9754$ & $\mathrm{NA}$ \\
\hline
\end{tabular}


Table XIV. Physical properties of "Gazipur - Brown" class

\begin{tabular}{l|c|c|c|c}
\hline $\begin{array}{c}\text { Egg sample/ } \\
\text { sample } \\
\text { statistics }\end{array}$ & $\begin{array}{c}\text { Property 1: } \\
\text { weight (g) }\end{array}$ & $\begin{array}{c}\text { USDAsize } \\
\text { grade }\end{array}$ & $\begin{array}{c}\text { Property 2: whole } \\
\text { weight ratio of raw to } \\
\text { complete boiled condition }\end{array}$ & $\begin{array}{c}\text { Property 3:boiled shell } \\
\text { albumen-yolk weight ratio }\end{array}$ \\
\hline $\mathrm{R}-1$ & 66.5640 & Extra large & $1: 0.9720$ & $1: 4.9691: 1.8388$ \\
$\mathrm{R}-2$ & 53.5825 & Medium & $1: 0.9745$ & $1: 3.9039: 1.7491$ \\
$\mathrm{R}-3$ & 64.4329 & Extra large & $1: 0.9732$ & $1: 4.9209: 1.6355$ \\
$\mathrm{R}-4$ & 65.5652 & Extra large & $1: 0.9730$ & $1: 5.2128: 1.8541$ \\
$\mathrm{R}-5$ & 64.9578 & Extra large & $1: 0.9728$ & $1: 4.4783: 1.7152$ \\
$\mathrm{R}-6$ & 59.3256 & Large & $1: 0.9712$ & $1: 5.3726: 1.8557$ \\
$\begin{array}{l}\text { Minimum } \\
\text { value }\end{array}$ & 53.5825 & Medium & $1: 0.9712$ & $1: 3.9039: 1.7491$ \\
$\begin{array}{l}\text { Maximum } \\
\text { value }\end{array}$ & 66.5640 & Extra large & $1: 0.9745$ & NA \\
Mean & 62.4047 & Large & $1: 0.9728$ & NA \\
$\begin{array}{l}\text { Standard } \\
\text { error }\end{array}$ & 2.0431 & NA & 0.0005 & 1.8557 \\
\hline
\end{tabular}

Table XV. Physical properties of "Gazipur - White" class

\begin{tabular}{l|c|c|c|c}
\hline $\begin{array}{c}\text { Egg sample/ } \\
\text { sample } \\
\text { statistics }\end{array}$ & $\begin{array}{c}\text { Property 1: } \\
\text { weight (g) }\end{array}$ & $\begin{array}{c}\text { USDA size } \\
\text { grade }\end{array}$ & $\begin{array}{c}\text { Property 2: whole } \\
\text { weight ratio of raw to } \\
\text { complete boiled condition }\end{array}$ & $\begin{array}{c}\text { Property 3: boiled shell } \\
\text { albumen-yolk weight ratio }\end{array}$ \\
\hline $\mathrm{R}-1$ & 58.4650 & Large & $1: 0.9706$ & $1: 5.0276: 2.1221$ \\
$\mathrm{R}-2$ & 58.4632 & Large & $1: 0.9715$ & $1: 4.5553: 1.9581$ \\
$\mathrm{R}-3$ & 57.8559 & Large & $1: 0.9718$ & $1: 4.9392: 1.9775$ \\
$\mathrm{R}-4$ & 57.7793 & Large & $1: 0.9735$ & $1: 3.6139: 1.5480$ \\
$\mathrm{R}-5$ & 63.6566 & Large & $1: 0.9695$ & $1: 3.9911: 1.6162$ \\
$\mathrm{R}-6$ & 62.8208 & Large & $1: 0.9716$ & $1: 4.3771: 1.5439$ \\
Minimum & 57.7793 & Large & $1: 0.9695$ & $1: 5.0276: 2.1221$ \\
value & & Large & $1: 0.9735$ & NA \\
$\begin{array}{l}\text { Maximum } \\
\text { value }\end{array}$ & 63.6566 & Large & $1: 0.9714$ & NA \\
$\begin{array}{l}\text { Mean } \\
\text { Standard } \\
\text { error }\end{array}$ & 59.8401 & NA & 0.0005 & \\
\hline
\end{tabular}


Table XVI. Physical properties of "Narayanganj - Brown" class

\begin{tabular}{l|c|c|c}
\hline $\begin{array}{c}\text { Egg sample/ } \\
\text { sample } \\
\text { statistics }\end{array}$ & $\begin{array}{c}\text { Property 1: } \\
\text { weight (g) }\end{array}$ & $\begin{array}{c}\text { USDA size } \\
\text { grade }\end{array}$ & $\begin{array}{c}\text { Property 2: whole } \\
\text { weight ratio of raw to complete } \\
\text { boiled condition }\end{array}$ \\
\hline $\mathrm{R}-1$ & 57.1362 & Large & $1: 0.9724$ \\
$\mathrm{R}-2$ & 57.9883 & Large & $1: 0.9698$ \\
$\mathrm{R}-3$ & 56.2474 & Medium & $1: 0.9740$ \\
$\mathrm{R}-4$ & 65.1325 & Extra Large & $1: 0.9712$ \\
$\mathrm{R}-5$ & 53.0277 & Medium & $1: 0.9753$ \\
$\mathrm{R}-6$ & 52.3909 & Medium & $1: 0.9682$ \\
$\mathrm{R}-7$ & 61.0535 & Large & $1: 0.9768$ \\
$\mathrm{R}-8$ & 61.1012 & Large & $1: 0.9705$ \\
$\mathrm{R}-9$ & 57.4035 & Large & $1: 0.9729$ \\
$\mathrm{R}-10$ & 61.6967 & Large & $1: 0.9689$ \\
$\mathrm{R}-11$ & 51.8180 & Medium & $1: 0.9699$ \\
$\mathrm{R}-12$ & 64.2368 & Extra large & $1: 0.9715$ \\
Minimum & & Medium & $1: 0.9682$ \\
value & 51.8180 & & $1: 0.9768$ \\
Maximum & & Extra large & $1: 0.9718$ \\
value & 65.1325 & Large & 0.0008 \\
Mean & 58.2694 & & NA \\
Standard & & & \\
error & 1.2912 & & \\
\hline
\end{tabular}

by a number of cytoplasmic reducing agents. The final cellular form of chromium, $\mathrm{Cr}$ (III), becomes trapped intracellularly because it has low cell membrane permeability. This shift from Cr (VI) to Cr (III) allows a concentration gradient to be established such that a continual influx of Cr (VI) ions raises intracellular chromium levels until lethal burdens are achieved. While both valence states of chromium are able to interact with DNA, Cr (III) ions are responsible for decreasing the fidelity of DNA replication. In addition, both $\mathrm{Cr}$ (III) and $\mathrm{Cr}$ (VI) exhibit a clastogenic potency; however, $\mathrm{Cr}$ (VI) possesses the greater activity and is also a powerful mutagen in many prokaryotic and eukaryotic cell systems. These properties of Cr (VI) support the claim that hexavalent compounds are likely to be active carcinogens, although it is more likely that the ultimate species responsible for the carcinogenic/mutagenic effects observed in vivo is the intracellularly derived trivalent form.

\section{Dietary reference intakes for chromium}

In 1989, the National Academy of Sciences established an "estimated safe and adequate daily dietary intake" range for chromium. For adults and adolescents that range was 50 to $200 \mu \mathrm{g}$ (NRC, 1989). In 2001, DRIs 
Table XVII. Physical properties of "Munshigonj - Brown" class

\begin{tabular}{l|c|c|c}
\hline $\begin{array}{c}\text { Egg sample/ } \\
\text { sample } \\
\text { statistics }\end{array}$ & $\begin{array}{c}\text { Property 1: } \\
\text { weight (g) }\end{array}$ & $\begin{array}{c}\text { USDA size } \\
\text { grade }\end{array}$ & $\begin{array}{c}\text { Property 2: whole } \\
\text { weight ratio of raw to complete boiled } \\
\text { condition }\end{array}$ \\
\hline $\mathrm{R}-1$ & 41.2333 & Peewee & $1: 0.9732$ \\
$\mathrm{R}-2$ & 37.8868 & Peewee & $1: 0.9697$ \\
$\mathrm{R}-3$ & 38.3685 & Peewee & $1: 0.9848$ \\
$\mathrm{R}-4$ & 40.4977 & Peewee & $1: 0.9809$ \\
$\mathrm{R}-5$ & 38.8135 & Peewee & $1: 0.9715$ \\
$\mathrm{R}-6$ & 38.8066 & Peewee & $1: 0.9711$ \\
$\mathrm{R}-7$ & 39.1801 & Peewee & $1: 0.9789$ \\
$\mathrm{R}-8$ & 38.9298 & Peewee & $1: 0.9733$ \\
$\mathrm{R}-9$ & 39.4001 & Peewee & $1: 0.9688$ \\
$\mathrm{R}-10$ & 41.2473 & Peewee & $1: 0.9926$ \\
$\mathrm{R}-11$ & 41.1069 & Peewee & $1: 0.9817$ \\
$\mathrm{R}-12$ & 44.0106 & Small & $1: 0.9915$ \\
Minimum & & & $1: 0.9688$ \\
value & 37.8868 & Peewee & $1: 0.9926$ \\
Maximum & 44.0106 & Small & $1: 0.9782$ \\
value & 39.9568 & Peewee & 0.0024 \\
Mean & & NA & \\
Standard & 0.4981 & & \\
error & & & \\
\hline
\end{tabular}

(Dietary Reference Intakes) for chromium were established. The research base was insufficient to establish RDAs (Recommended Dietary Allowances), so AIs (Adequate Intakes) were developed based on average intakes of chromium from food as found in several studies (Institute of Medicine, 2001). Chromium AIs are provided in Table IXX.

\section{Tolerable upper intake level (UL)}

Few serious adverse effects have been linked to high intakes of chromium, so the Institute of Medicine has not established a Tolerable Upper Intake Level (UL) for this mineral (Stoecker, 2001; Institute of Medicine, 2001). A UL is the maximum daily intake of a nutrient that is unlikely to cause adverse health effects. It is one of the values [together with the RDA (Recommended Dietary Allowance) and AI (Adequate Intakes)] that comprise the Dietary Reference Intakes (DRIs) for each nutrient.

\section{Assessment of tannery based eco-toxicity}

Chromium is quite variable among different lots of foods (Anderson et al., 1992) and may be influenced by geochemical factors (Welch and Cary, 1975). One of the direct ways of the tannery based chromium ecotoxicity in Bangladesh should be its bio-concentration into poultry eggs. Out of the 192 samples from 96 eggs, dry weight basis 


\begin{tabular}{|c|c|c|c|c|c|c|c|c|c|c|c|}
\hline & 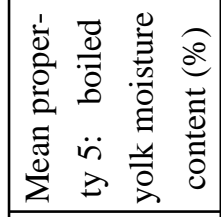 & 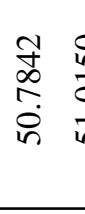 & 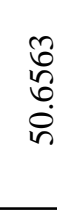 & 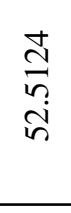 & $\begin{array}{l}\vec{\nabla} \\
\text { m. } \\
\dot{\vec{n}}\end{array}$ & ' & ' & ' & & ' & ' \\
\hline & 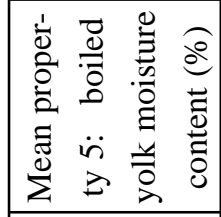 & 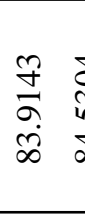 & $\begin{array}{l}\text { D్ } \\
\stackrel{0}{+} \\
\dot{\Phi}\end{array}$ & $\begin{array}{l}\stackrel{\infty}{0} \\
\hat{b} \\
\tilde{m} \\
\dot{\infty}\end{array}$ & $\begin{array}{l}\mathscr{0} \\
\infty \\
\infty \\
\infty \\
\infty\end{array}$ & ' & ' & ' & ' & ' & ' \\
\hline & 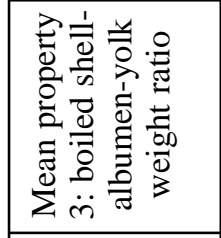 & 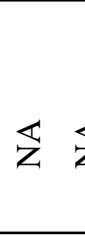 & $\overleftrightarrow{Z}$ & $\overleftrightarrow{Z}$ & 艺 & Z & $\overleftrightarrow{Z}$ & 飞 & $\mathbb{Z}$ & ' & ' \\
\hline : & 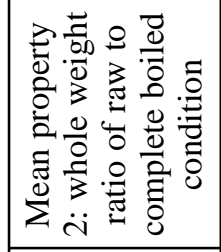 & 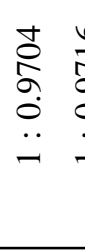 & 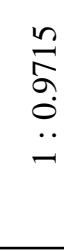 & 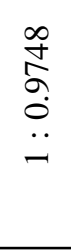 & 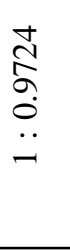 & $\begin{array}{l}\stackrel{+}{A} \\
\hat{\sigma} \\
0 \\
\ddot{-} \\
-\end{array}$ & 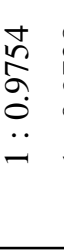 & 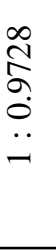 & 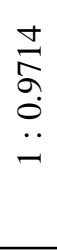 & - & 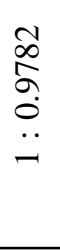 \\
\hline 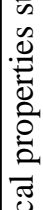 & 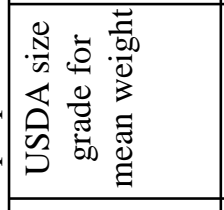 & 恋 & 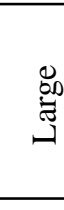 & $\begin{array}{l}\text { ه్ } \\
\text { 亗 }\end{array}$ & 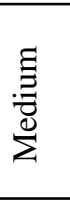 & $\begin{array}{l}\text { 罵 } \\
\underbrace{\circ}_{\Sigma}\end{array}$ & $\begin{array}{l}\stackrel{\circ}{\infty} \\
\stackrel{\varpi}{\oplus}\end{array}$ & 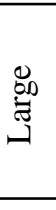 & 兽 & & 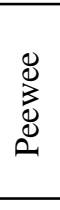 \\
\hline$\frac{\substack{n \\
\frac{n}{2}}}{2}$ & 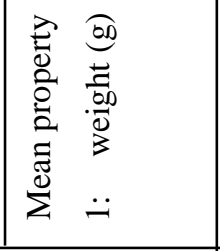 & $\begin{array}{l}\text { هे } \\
\text { oे } \\
\stackrel{0}{0} \\
\text { î }\end{array}$ & $\begin{array}{l}\mathscr{0} \\
\mathbb{0} \\
0 \\
0\end{array}$ & 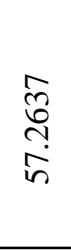 & $\begin{array}{l}\stackrel{+}{+} \\
\infty \\
\infty \\
\dot{H}\end{array}$ & $\begin{array}{l}\text { 员 } \\
\infty \\
\text { กิ } \\
\text { ஸे }\end{array}$ & 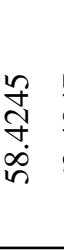 & 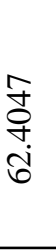 & 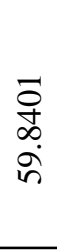 & & $\begin{array}{l}\infty \\
0 \\
\swarrow \\
\sigma \\
\infty \\
\infty\end{array}$ \\
\hline & $\begin{array}{l}\text { 常 } \\
\text { 空 } \\
\text { 心 }\end{array}$ & 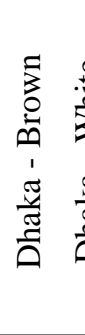 & 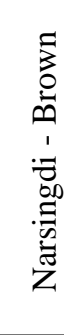 & 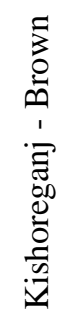 & 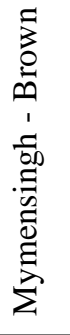 & 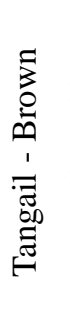 & 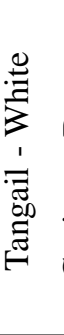 & 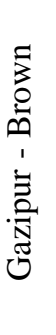 & 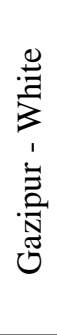 & & 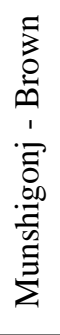 \\
\hline & 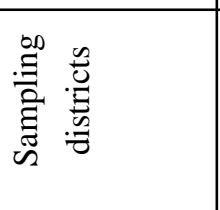 & $\begin{array}{l}\frac{\pi}{\pi} \\
\frac{\pi}{\pi}\end{array}$ & 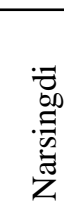 & 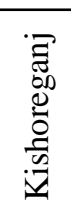 & 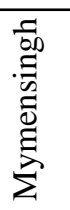 & 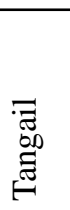 & & & & & 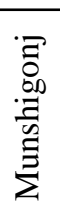 \\
\hline
\end{tabular}


Table IXX. Adequate Intakes (AIs) for chromium (Institute of Medicine, 2001)

\begin{tabular}{l|c|c|c|c|c}
\hline \multicolumn{1}{c|}{ Age } & $\begin{array}{c}\text { Infants and chil- } \\
\text { dren }(\mu \mathrm{g} / \mathrm{day})\end{array}$ & $\begin{array}{c}\text { Males } \\
(\mu \mathrm{g} / \mathrm{day})\end{array}$ & $\begin{array}{c}\text { Females } \\
(\mu \mathrm{g} / \mathrm{day})\end{array}$ & $\begin{array}{c}\text { Pregnancy } \\
(\mu \mathrm{g} / \mathrm{day})\end{array}$ & $\begin{array}{c}\text { Lactation } \\
(\mu \mathrm{g} / \mathrm{day})\end{array}$ \\
\hline 0 to 6 months & 0.2 & & & & \\
7 to 12 months & 5.5 & & & & \\
1 to 3 years & 11 & & & & \\
4 to 8 years & 15 & 25 & 21 & & \\
9 to 13 years & & 35 & 24 & 29 & 44 \\
14 to 18 years & & 35 & 25 & 30 & \\
19 to 50 years & & 30 & 20 & & \\
$>50$ years & & & & & \\
\hline
\end{tabular}

mean chromium concentration was found to be $1.9016 \mathrm{ppm}$ with a sample standard deviation of 0.1502 . The concentration levels were ranged from maximum of $19.8051 \mathrm{ppm}$ to undetectable levels. From the data, it is clearly seen that the mean chromium content of a single egg is $23.3809 \mu$ g, which exceeds adequate daily dietary intake of children up to 8 years of age as well as corresponds to major part for other age groups. As the Institute of Medicine, USA has not established a Tolerable Upper Intake Level (UL) for chromium due to its adverse health effects observed in cases of high intakes, any intake exceeding the adequate daily dietary intake level is being considered undesirable. If a single poultry egg can contain more than the amount which should be taken from all dietary intakes in one day, then along with the chromium present in other food items consumed in a day must turn the total amount exceeding the safety limit, especially for the children.
An important fact has been observed that in all sample classes except Gazipur white eggs, the chromium content is higher in albumen than in yolk. From these results, it can be suggested that albumen is more likely to contain chromium (total chromium, irrespective of the valence states) than do yolk for chicken eggs so far as bio-concentration is concerned.

\section{Discussion on region-wise physical properties of the eggs}

From Table XVIII, the mean whole weights of eggs at raw condition were within the range of $39.9568 \mathrm{~g}$ ('peewee' as per USDA size grading) to $62.4047 \mathrm{~g}$ ('large' as per USDA size grading). All the sample classes have 'medium' or 'large' mean USDA size grade except eggs from Munshigonj which had a mean size grade of 'pewee'. The mean whole weight ratio of raw condition to complete boiled condition ranged between "1: $0.9704 "$ to "1: $0.9782 "$, which has a very close interval. From the data presented in Table VII to Table XVII the minimum weight 
ratio of boiled shell-albumen-yolk for all sample classes were ranged from " $1: 2.0714$ : $1.1549 "$ to "1 : 4.8810 : 2.0677" whereas the maximum weight ratio ranged from "1 : $3.1933: 1.4557 "$ to " $1: 7.8084: 3.3818 "$. In this case, the ranges were quite large reflecting the inherent variability of chicken eggs within and among sample classes. The mean moisture content of albumen of boiled eggs varied within the range of $82.8876 \%$ to $84.7636 \%$, and the mean moisture content of yolk of boiled eggs were between 50.6563 $\%$ to $52.5124 \%$. Though not very wide, these ranges have shown a fairly constant property for all sampled eggs.

\section{Conclusion}

A number of facts can be of special importance regarding the findings. It can be likely that, as the exact routes of distribution of largely produced protein-concentrates from SCW are unknown, the affected areas may not be reflected in the areas selected for study. But as the sampling districts comprise the central region of Bangladesh, the tannery based chromium eco-toxicity can be quantitatively described for commercially produced chicken eggs with the results obtained for the mentioned region. Again, as there is no set Tolerable Upper Intake Level (UL) for chromium, as well as the less toxic $\mathrm{Cr}$ (III) or the intermediates during reduction of $\mathrm{Cr}(\mathrm{VI})$ can be the ultimately responsible species for carcinogenicity, the results obtained for the selected region obviously carry importance, especially in regard to adequate daily dietary intake. But specific researches with the controlled application of the SCW produced protein-concentrates can exactly reveal the potency of the product for the bio-accumulation of chromium in chicken body and eggs. Research on the inventory of distribution routes of the product throughout Bangladesh will also help significantly to assess its ecotoxicity phenomenon. Besides, the inventory of physical properties will be of importance for local-level as well as basic researches on poultry.

\section{References}

Anderson, R.A., Bryden, N.A. Polansky, M.M. (1992). Dietary chromium intake. Freely chosen diets, institutional diets, and individual foods. Biol Trace Elem Res 32: 117-121.

Baruthio, F. (1992). Toxic effects of chromium and its compounds; Biol. Trace Elem. Res., 32: $145-53$.

BBS. (2004). Statistical yearbook of Bangladesh. (Bangladesh Bureau of Statistics, Government of People's Republic of Bangladesh).

BRAC. (2005). BRAC Report on Poultry Farms 2005. (BRAC, Dhaka, Bangladesh).

Clark, D.P. (1994). Chromate reductase activity of Enterobacter aerogenus is induced by nitrite. FEMS Microbiol. Lett., 122: 233-238.

Cohen, M.D. Costa, M. (2000). Chromium. pp. 173-191. In Morton Lippmann (ed.) Environmental Toxicants: Human Exposures 
and Their Health Effects, 2/e. (John Wiley and Sons, Inc).

FAO. (2007a).(http://www.fao.org/ag/AGAinfo/ subjects/en/eggs.html).

FAO. (2007b). (http://www.fao.org/AG/againfo/ subjects/en/subjects.html).

Gillin, Edward. (2003). World Egg and Poultry meat Production, Trade, and Supply: Present and the Future. (www.fao.org/ag/againfo/ subjects/documents/eggs/Egg-PoultryProduction.pdf).

Greenwood, N.N. Earnshaw, A. (1997). Chemistry of the Elements, 2/e. (Oxford: Butterworth-Heinemann).

Hossain, A.M.M.M., Monir, T. Rezwan - Ul Haque, A.M. Kazi, M.A.I. Islam, M.S. Elahi, S.F. (2007). Heavy metal concentration in tannery solid wastes used as poultry feed and the ecotoxicological consequences. Bangladesh J. Sci. Ind. Res., 42(4): 397-416.

Institute of Medicine, Food and Nutrition Board. (2001). Dietary Reference Intakes for Vitamin A, Vitamin K, Arsenic, Boron, Chromium, Copper, Iodine, Iron, Manganese, Molybdenum, Nickel, Silicon, Vanadium, and Zinc. (National Academy Press, Washington, DC).

Kebbekus, B.B. Mitra, S. (1998). Environ-mental Chemical Analysis. (Blackie Academic \& Professional, London).

Kerger, B.D., Finley, B.L. Corbett, G.E. Dodge, D.G. Paustenbach, D.J. (1997). Ingestion of
chromium(VI) in drinking water by human volunteers: Absorption, distribution, and excretion of single and repeated doses. $J$. Toxicol. Environ. Health, 50: 67-95.

Kuykendall, J.R., Kerger, B.D. Jarvi, E.J. Corbett, G.E. Paustenbach, D. J. (1996). Measurement of DNA-protein cross-links in human leukocytes following acute ingestion of chromium in drinking water. Carcinogenesis, 17: 1971-1977.

McLean, J. Beveridge, T.J. (2001). Chromate reduction by a pseudomonad isolated from a site contaminated with chromated copper arsenate. Appl. Environ. Microbiol., 67: 1076-1084.

Mertz, W. (1998). Interaction of chromium with insulin: a progress report. Nutr. Rev., 56: 174-7.

NRC. (1980). Mineral Tolerance of Domestic Animals. (National Academy Press, Washington, DC).

National Research Council, Food and Nutrition Board. (1989). Recommended Dietary Allowances, 10/e. (National Academy Press, Washington, DC).

Poultry business Directory. (2007). Poultry Business Directory 2007. (Poultry Khamar Bichitra, Dhaka, Bangladesh).

Rahman, M. (2007). Poverty Alleviation and Employment Opportunity through Poultry. (5th International Poultry Show and Seminar 2007, WPSA-BB, Dhaka, Bangladesh). 
Stoecker, B.J. (2001). Chromium. pp. 366-372. In Bowman, B., and R. Russell (ed) Present Knowledge in Nutrition, 8/e. (ILSI Press, Washington, DC).

UNIDO. (2000). Regional Programme for Pollution Control in the Tanning Industry in South-East Asia: Chrome Balance in Leather Processing. (United Nations Industrial Development Organization, Vienna, Austria).

USDA. (2000). Egg Grading Manual. (United States Department of Agriculture, www.ams.usda.gov/Poultry/pdfs/EggGradin g\%20manual.pdf).
Welch, R.M. Cary, E.E. (1975). Concentra-tion of chromium, nickel, and vanadium in plant materials. J. Agric. Food Chem., 23: 479482.

Zahid, A., Balke, K.D., Hassan, M.Q. Flegr, M. (2004): Distribution of heavy metals in tannery effluent and their influence on sediments of Hazaribagh leather processing zone, Dhaka. In M. Q. Hassan (ed.) Water Resources Management and Development in Dhaka City. (Goethe-Institut Dhaka).

Received :January 01, 2008;

Accepted : June, 23, 2008. 